\section{Pulmonary embolism in old age: usefulness of risk stratification in clinical decision-making}

\author{
Carlo Bova, Vitaliano Spagnuolo, \\ Alfonso Noto \\ Department of Internal Medicine, \\ Annunziata General Hospital, Cosenza, \\ Italy
}

\section{Abstract}

Pulmonary embolism (PE) is a common disease with a not negligible short-term risk of death, in particular in the elderly. An adequate evaluation of the prognosis in patients with $\mathrm{PE}$ may guide decision-making in terms of the intensity of the initial treatment during the acute phase. Patients with shock or persistent hypotension are at high risk of early mortality and may benefit from immediate reperfusion. Several tools are available to define the shortterm prognosis of hemodynamically stable patients. The pulmonary embolism severity index (PESI) score, and the simplified PESI score are particularly useful for identifying patients at low risk of early complications who might be safely treated at home. The identification of patients who are hemodynamically stable at diagnosis but are at a high risk of early complications is more challenging. Current guidelines recommend a multi-parametric prognostic algorithm based on the clinical status, biomarkers and imaging tests. However an aggressive treatment in hemodynamically stable patients is still controversial.

\section{Case Report}

A 83-year-old woman without a significant medical history, and recent surgery because of traumatic hip fracture, presented to the emergency department of our hospital with sudden onset of right-sided chest discomfort and dyspnea that occurred during rehabilitation treatment in a long-term care facility. On physical examination, she was tachycardic (heart rate 116 beats per minute), normotensive (blood pressure of 100/72 mm $\mathrm{Hg}$ ), tachypneic (22 breaths per minute), and hypoxemic (oxygen saturation $87 \%$ on room air). She had jugular venous distension, lung fields clear to auscultation bilaterally, and mild right lower-extremity edema. The echocardiography (ECG) was notable for sinus tachycardia and T-wave inversions across the anterior precordial leads. Laboratory evaluation was remarkable for a cardiac troponin I level of $0.9 \mathrm{ng} / \mathrm{mL}$ (normal $\leq 0.04 \mathrm{ng} / \mathrm{mL}$ ). Bedside transthoracic ECG documented a right ventricular (RV) enlargement (RV-to-left ventricular dimension ratio 1.5). Contrast-enhanced chest computed tomography (CT) demonstrated a thrombus that filled the right main pulmonary artery. How should this patient be managed?

\section{Discussion}

Acute pulmonary embolism (PE) is a potentially life-threatening condition, being the third cause of death among the cardiovascular diseases. ${ }^{1}$ Elderly patients are not only at particularly high risk for PE, but also at high risk of adverse clinical outcomes and treatment-related complications. ${ }^{2}$ PE has a wide range of clinical presentations, that are associated with different clinical severity and risk for adverse short-term outcomes. ${ }^{3}$ Patients with persistent arterial hypotension $(<90 \mathrm{~mm}$ $\mathrm{Hg}$ ) or cardiogenic shock represent only about $5 \%$ of all patients, but have a high risk of early death. ${ }^{3}$ Patients with normal blood pressure have usually a good prognosis when properly anticoagulated, with a mortality rate as low as $2 \% .^{4}$ Some subgroups may however experience a less favorable course. ${ }^{5}$ Therefore, a risk stratification in pulmonary embolism is widely deemed necessary to guide patient management.

\section{Hemodynamic status}

The hemodynamic status is the most important prognostic criterion. In the International cooperative pulmonary embolism registry, patients with massive pulmonary embolism, defined as systolic arterial pressure $<90 \mathrm{~mm}$ $\mathrm{Hg}$, had a $54 \%$ mortality at 90 days, versus a mortality rate of $15 \%$ in normotensive patients. ${ }^{6}$

\section{Clinical scores}

Several clinical prediction models have been developed to determine the outcome of patients with PE. Of these, the pulmonary embolism severity index (PESI) and a simplified version, sPESI, have been the most extensively validated (Table 1), ${ }^{7,8}$ The principal strength of the PESI and sPESI lies in the reliable identification of patients at low risk for 30-day mortality (PESI Class I and II or sPESI Class 0). For example, a score 0 in the sPESI is associated with a mortality rate of $1 \%$ at 30 days. By contrast, a sPESI of 1 predicts a 30-day risk of death of $11 \%$. Instead, the usefulness of these scores in guiding the choice of more aggressive therapy (for example, thrombolysis) when they are positive is uncertain. Indeed, these scores provide the risk of all-cause death, which can be due to the co-morbidities included, regard-
Correspondence: Carlo Bova, Department of Internal Medicine, Annunziata General Hospital, via Migliori 1, 87100 Cosenza, Italy.

Tel/Fax: +39.0984 .681401$

E-mail: carbova@libero.it

Key words: Pulmonary embolism; risk-stratification; mortality.

Contributions: $\mathrm{CB}$, study concept and design and drafting of the manuscript; $\mathrm{CB}, \mathrm{VS}, \mathrm{AN}$, acquisition of data, analysis and interpretation of data, critical revision of the manuscript for important intellectual content. All authors approved the final version of the article.

Conflict of interest: the authors declare no potential conflict of interest.

Received for publication: 11 June 2016.

Revision received: 16 August 2016.

Accepted for publication: 22 August 2016.

This work is licensed under a Creative Commons Attribution-NonCommercial 4.0 International License (CC BY-NC 4.0)

(c) Copyright C. Bova et al., 2016

Licensee PAGEPress, Italy

Geriatric Care 2016; 2:6080

doi:10.4081/gc.2016.6080

less of the risk due to PE. One randomized trial employed a low PESI as the inclusion criterion for home treatment of PE.

\section{Age}

Elderly patients with acute pulmonary embolism have higher in-hospital mortality than younger patients. ${ }^{10}$ Older age is included as prognostic factor in both PESI and sPESI. In this latter even the single criterion of age $>80$ years is associated with an sharp increase in short-term mortality. ${ }^{8}$

\section{Right ventricular dysfunction}

Right ventricular dysfunction (RVD) is identified by ECG or computed tomographic angiography in $25-50 \%$ of patients with PE. ${ }^{11}$ RVD is a recognized risk factor for adverse events in patients with PE. In PE patients without hemodynamic compromise, RVD, as assessed by ECG or CT, was associated with a risk ratio for all-cause mortality of 2.4 [ $95 \%$ confidence interval (CI) 1.3-4.4]. ${ }^{11}$ However, its positive predictive value (PPV) for all-cause or PE-related death was low (5-8\%). Several, non-standardized, criteria have been used to diagnose RVD with ECG, such as right-to-left ventricular ratio, right ventricular hypokinesis, and elevated systolic pulmonary artery pressure. ${ }^{12,13}$ The only CT criterion for RVD is the right-to-left ventricular ratio. ${ }^{14}$ 


\section{Cardiac biomarkers}

A number of cardiac biomarkers have been shown to be associated with adverse events in PE, including troponins, brain-type natriuretic peptide and fatty acid-binding protein. ${ }^{15,16}$ In one meta-analysis troponins predicted allcause mortality in hemodynamically stable patients with PE (odds ratio 5.90; 95\% CI, 2.6812.95). ${ }^{15}$ However, in another meta-analysis elevated troponin levels did not adequately discern normotensive patients who were at a high risk of death from those who are at low risk. ${ }^{16}$

Given the well-recognized worse prognosis in hemodynamically unstable patients with PE, the prognostic usefulness of imaging tests or biomarkers to guide therapeutic decisions is better evaluated in hemodynamically stable PE patients. However, neither RVD nor abnormal biomarkers, as a single prognostic criterion, have sufficient PPV for PE-related adverse events to guide therapeutic decisions in normotensive patients with PE. ${ }^{5}$

\section{Combined risk factors}

With the aim to improve the PPV for adverse events in PE, several scores combining multiple risk-factors have been proposed. ${ }^{17}$ Among them, the most extensively validated is the Bova score (Table 2). ${ }^{18,19}$ Recently, an algorithm for the risk-stratification of patients with PE has been proposed by the European Society of Cardiology (ESC) (Table 3). ${ }^{17}$

\section{Role of thrombolytic therapy in pulmonary embolism}

Thrombolytics can be life saving in hemodynamically unstable patients with $\mathrm{PE}$, and current guidelines suggest their use in this situation. ${ }^{17}$ However, in normotensive patients, the usefulness of these drugs is controversial. In the recent large Pulmonary Embolism Thrombolysis trial $^{20} 1005$ normotensive patients with right ventricular dysfunction on ECG or CT, and a positive test for cardiac troponin, were randomized to tenecteplase plus heparin or placebo plus heparin. Death or hemodynamic decompensation occurred in $2.6 \%$ of patients of the tenecteplase group and in $5.6 \%$ of the placebo group $(\mathrm{P}=0.02)$. There were no differences in mortality rate. The risk of major hemorrhage and stroke was signifi- cantly increased in the tenecteplase group. Older patients ( $>75$ years) treated with tenecteplase had a not significant higher rate of major extracranial bleeding than did younger patients $(\mathrm{P}=0.09)$. Interestingly, in two small studies a strategy using a halveddose of tissue plasminogen activator (rtPA) appeared to be safe compared with anticoagulation alone, while maintaining similar efficacy than usual dose. ${ }^{12,13}$ Although these studies did not focus on elderly (mean age 58 and 52 years respectively), their results are appealing for older patients, usually at higher risk of bleeding, and might empirically guide thrombolytic therapy (when deemed appropriate) in these patients. Current guidelines do not recommend thrombolytic therapy in hemodynamically stable patients with PE. ${ }^{17}$

\section{Conclusions}

In the case presented here, the patient met the criteria for the intermediate-high risk class of the ESC classification, and for the third stage of the Bova score. According to this latter, the patient had a risk of about $29-37 \%$ for short-term adverse events PE-related. ${ }^{18,19}$ Anticoagulation was started with enoxaparin 1 $\mathrm{mg} / \mathrm{kg}$ subcutaneously. One hour later, the arterial pressure fell to $60 / 40 \mathrm{~mm} \mathrm{Hg}$, and signs of peripheral hypo-perfusion manifested. A halved dose of rtPA ( $50 \mathrm{mg}$ ) was administered intravenously (a bolus of $5 \mathrm{mg}$ followed by infusion in two hours). Thirty minutes after the start of infusion the arterial pressure began to increase and the dyspnea abated, and two hours later the patient felt well. No side effects occurred. A follow-up one-month later showed an excellent health status.

\section{References}

1. Lloyd-Jones D, Adams RJ, Brown TM, et al. Heart disease and stroke statistics-2010 update: a report from the American Heart Association. Circulation 2010;121:e46$\mathrm{e} 215$.

Table 1. Simplified pulmonary embolism severity index.

\begin{tabular}{lc} 
Variable & Score \\
Age $>80$ years & 1 \\
History of cancer & 1 \\
\hline History of chronic cardiopulmonary disease & 1 \\
Heart rate $\geq 110$ & 1 \\
\hline Systolic blood pressure $<100 \mathrm{~mm} \mathrm{Hg}$ & 1 \\
Arterial oxyhemoglobin saturation $<90 \%$ & 1 \\
\hline
\end{tabular}

Table 2. Bova score.

\begin{tabular}{lc} 
Predictor & Points * \\
SBP $90-100 \mathrm{~mm} \mathrm{Hg}$ & 2 \\
Elevated cardiac troponin & 2 \\
\hline RVD (echocardiogram or CT scan) & 2 \\
Heart rate >110/min & 1 \\
\hline SBP, systolic blood pressure; RVD, right ventricle dysfunction; CT, computed tomography. *Points $0-2$, stage I; 3-4, stage II; >4, stage III. \\
Percentage of patients: 75.5 stage I; 18.6 stage II; 5.8 stage III. 30-day pulmonary embolism-related complications (\%): 4.2 stage I; 10.8 stage II; \\
29.2 stage III.
\end{tabular}

Table 3. European Society of Cardiology algorithm for risk stratification in pulmonary embolism.

\begin{tabular}{|c|c|c|c|c|}
\hline & Shock or hypotension & PESI class III-IV or sPESI $\geq 1$ & Signs of RVD & Cardiac biomarkers \\
\hline High & + & NA & NA & NA \\
\hline Intermediate-high & - & + & + & + \\
\hline Intermediate-low & - & + & \multicolumn{2}{|c|}{ Either one (or none) positive } \\
\hline Low & - & - & \multicolumn{2}{|c|}{ Assessment optional; if assessed, both negative } \\
\hline
\end{tabular}


2. Robert-Ebadi H, Righini M. Diagnosis and management of pulmonary embolism in the elderly. Eur J Intern Med 2014;25:343-9.

3. Goldhaber SZ, Visani L, De Rosa M. Acute pulmonary embolism: clinical outcome in the International Cooperative Pulmonary Embolism Registry. Lancet 1999;353:1386-9.

4. Pollack CV, Schreiber D, Goldhaber SZ, et al. Clinical characteristics, management, and outcomes of patients diagnosed with acute pulmonary embolism in the emergency department: initial report of EMPEROR (Multicenter Emergency Medicine Pulmonary Embolism in the Real World Registry). J Am Coll Cardiol 2011;57:700-6.

5. Bova C, Pesavento R, Marchiori A, et al. Risk stratification and outcomes in hemodynamically stable patients with acute pulmonary embolism: a prospective, multicentre, cohort study with three months of follow-up. J Thromb Haemost 2009;7:938-44.

6. Kucher N, Rossi E, De Rosa M. Massive pulmonary embolism. Circulation 2006; 113:577-82.

7. Aujesky D, Obrosky DS, Stone RA. Derivation and validation of a prognostic model for pulmonary embolism. Am J Respir Crit Care Med 2005;172:1041-6.

8. Jimenez D, Aujesky D, Moores L. Simplification of the pulmonary embolism severity index for prognostication in patients with acute symptomatic pulmonary embolism. Arch Intern Med 2010;170:1383-9.

9. Aujesky D, Roy PM, Verschuren F. Outpatient versus inpatient treatment for patients with acute pulmonary embolism: an international, open-label, randomized, non-inferiority trial. Lancet 2011;378:41-8.

10. Punukollu H, Khan IA, Punukollu G. Acute pulmonary embolism in elderly: clinical characteristics and outcome. Int J Cardiol. 2005;99:213-6.

11. Sanchez 0, Trinquart L, Colombet I. Prognostic value of right ventricular dysfunction in patients with haemodynamically stable pulmonary embolism: a systematic review. Eur Heart J 2008;29:1569-77.

12. Sharifi M, Bay C, Skrocki L. Moderate pulmonary embolism treated with thrombolysis (from the "MOPETT" Trial). Am J Cardiol 2013;111:273-7.

13. Wang $C$, Zhai Z, Yang Y. Efficacy and safety of low dose recombinant tissue-type plasminogen activator for the treatment of acute pulmonary thromboembolism: a randomized, multicenter, controlled trial. Chest 2010;137:254-62.

14. Trujillo-Santos J, den Exter PL, Gómez V. Computed tomography assessed right ventricular dysfunction and risk stratification of patients with acute non-massive pulmonary embolism: systematic review and meta-analysis. J Thromb Haemost 2013; 11:1823-32.

15. Becattini C, Vedovati MC, Agnelli G. Prognostic value of troponins in acute pulmonary embolism: a meta-analysis. Circulation 2007;116:427-33.

16. Jimenez D, Uresandi F, Otero R. Troponinbased risk stratification of patients with acute nonmassive pulmonary embolism: systematic review and meta-analysis. Chest 2009;136:974-82.

17. Konstantinides SV, Torbicki A, Agnelli G; 2014 ESC guidelines on the diagnosis and management of acute pulmonary embolism. Eur Heart J 2014;35:3033-69, 3069a-3069k.

18. Bova C, Sanchez 0, Prandoni P. Identification of intermediate-risk patients with acute symptomatic pulmonary embolism. Eur Respir J 2014;44:694-703.

19. Fernández C, Bova C, Sanchez O. Validation of a model for identification of patients at intermediate to high risk for complications associated with acute symptomatic pulmonary embolism. Chest 2015;148:211-8.

20. Meyer G, Vicaut E, Danays T, et al. Fibrinolysis for patients with intermediate-risk pulmonary embolism. $\mathrm{N}$ Engl $\mathrm{J}$ Med 2014;370:1402-11. 